\title{
Positive Solutions for a Class of Singular Boundary Value Problems with Fractional $q$-Difference Equations
}

\author{
Jufang Wang, Changlong Yu, and Yanping Guo \\ College of Sciences, Hebei University of Science and Technology, Shijiazhuang, Hebei 050018, China \\ Correspondence should be addressed to Changlong Yu; changlongyu@126.com
}

Received 6 July 2014; Accepted 21 September 2014

Academic Editor: Kishin Sadarangani

Copyright (C) 2015 Jufang Wang et al. This is an open access article distributed under the Creative Commons Attribution License, which permits unrestricted use, distribution, and reproduction in any medium, provided the original work is properly cited.

We discuss a class of singular boundary value problems of fractional $q$-difference equations. Some existence and uniqueness results are obtained by a fixed point theorem in partially ordered sets. Finally, we give an example to illustrate the results.

\section{Introduction}

In recent years, many papers on fractional differential equations have appeared, because of their demonstrated applications in various fields of science and engineering; see [1-11] and the references therein. Based on the increasingly extensive application of discrete fractional calculus and the development of $q$-difference calculus or quantum calculus (see [12-19] and the references therein), fractional $q$-difference equations have attracted the attention of researchers for the numerous applications in a number of fields such as physics, chemistry, aerodynamics, biology, economics, control theory, mechanics, electricity, signal and image processing, biophysics, blood flow phenomena, aerodynamics, and fitting of experimental data; see [20-23]. Some recent work on the existence theory of fractional $q$-difference equations can be found in [24-29]. However, the study of singular boundary value problems (BVPs) with fractional $q$-difference equations is at its infancy and lots of work on the topic should be done.

Recently, in [25], Ferreira has investigated the existence of positive solution for the following fractional $q$-difference equations BVP

$$
\begin{gathered}
\left(D_{q}^{\alpha} y\right)(x)+f(x, y(t))=0, \quad 0<x<1, \\
y(0)=\left(D_{q}\right) y(0)=0, \quad\left(D_{q}\right) y(1)=\beta \geq 0,
\end{gathered}
$$

by applying a fixed point theorem in cones.
More recently, in [30], Caballero et al. have studied positive solutions for the following BVP:

$$
\begin{gathered}
\left(D_{0^{+}}^{\alpha} u\right)(t)+f(t, u(t))=0, \quad 0<t<1, \\
u(0)=u(1)=0,
\end{gathered}
$$

by fixed point theorem in partially ordered sets.

Motivated by the work above, in this paper, we discuss the existence and uniqueness of solutions for the singular BVPs of factional $q$-difference equations given by

$$
\begin{gathered}
\left(D_{q}^{\alpha} u\right)(t)+f(t, u(t))=0, \quad 0<t<1, \\
u(0)=u(1)=0, \quad\left(D_{q} u\right)(0)=0,
\end{gathered}
$$

where $2<\alpha \leq 3$ and $f:[0,1] \times[0, \infty) \rightarrow[0, \infty)$ is continuous with $\lim _{t \rightarrow 0^{+}} f(t, \cdot)=\infty$ (i.e., $f$ is singular at $t=0)$.

\section{Preliminary Results}

For convenience, we present some definitions and lemmas which will be used in the proofs of our results.

Let $q \in(0,1)$ and define

$$
[a]_{q}=\frac{1-q^{a}}{1-q}, \quad a \in R
$$



is

The $q$-analogue of the power function $(a-b)^{n}$ with $n \in N_{0}$

$$
\begin{array}{r}
(a-b)^{(0)}=1, \quad(a-b)^{(n)}=\prod_{k=0}^{n-1}\left(a-b q^{k}\right), \\
n \in N, \quad a, b \in R .
\end{array}
$$

More generally, if $\alpha \in R$, then

$$
(a-b)^{(\alpha)}=a^{\alpha} \prod_{n=0}^{n-1} \frac{a-b q^{n}}{a-b q^{\alpha+n}}
$$

Note that if $b=0$ then $a^{(\alpha)}=a^{\alpha}$. The $q$-gamma function is define by

$$
\Gamma_{q}(x)=\frac{(1-q)^{(x-1)}}{(1-q)^{x-1}}, \quad x \in R \backslash\{0,-1,-2, \ldots\},
$$

and it satisfies $\Gamma_{q}(x+1)=[x]_{q} \Gamma_{q}(x)$. [12].

Following, let us recall some basic concepts of $q$-calculus

Definition 1. For $0<q<1$, we define the $q$-derivative of a real-value function $f$ as

$$
\begin{aligned}
& \left(D_{q} f\right)(x)=\frac{f(x)-f(q x)}{(1-q) x}, \\
& \left(D_{q} f\right)(0)=\lim _{x \rightarrow 0}\left(D_{q} f\right)(x) .
\end{aligned}
$$

Note that $\lim _{q \rightarrow 1^{-}} D_{q} f(x)=f^{\prime}(x)$.

Definition 2. The higher order $q$-derivatives are defined inductively as

$$
\begin{gathered}
\left(D_{q}^{0} f\right)(x)=f(x) \\
\left(D_{q}^{n} f\right)(t)=D_{q}\left(D_{q}^{n-1} f\right)(t), \quad n \in N
\end{gathered}
$$

For example, $D_{q}\left(t^{k}\right)=[k]_{q} t^{k-1}$, where $k$ is a positive integer and the bracket $[k]_{q}=\left(q^{k}-1\right) /(q-1)$. In particular, $D_{q}\left(t^{2}\right)=(1+q) t$.

Definition 3. The $q$-integral of a function $f$ in the interval $[0, b]$ is given by

$$
\begin{array}{r}
\left(I_{q} f\right)(x)=\int_{0}^{x} f(t) d_{q} t=x(1-q) \sum_{n=0}^{\infty} f\left(x q^{n}\right) q^{n}, \\
x \in[0, b] .
\end{array}
$$

If $a \in[0, b]$ and $f$ is defined in the interval $[0, b]$, its integral from $a$ to $b$ is define by

$$
\int_{a}^{b} f(t) d_{q} t=\int_{0}^{b} f(t) d_{q} t-\int_{0}^{a} f(t) d_{q} t
$$

Similarly as done for derivatives, an operator $I_{q}^{n}$ can be define, namely,

$$
\begin{gathered}
\left(I_{q}^{0} f\right)(x)=f(x), \\
\left(I_{q}^{n} f\right)(x)=I_{q}\left(I_{q}^{n-1} f\right)(x), \quad n \in N .
\end{gathered}
$$

Observe that

$$
D_{q} I_{q} f(x)=f(x),
$$

and if $f$ is continuous at $x=0$, then $I_{q} D_{q} f(x)=f(x)-f(0)$.

We now point out three formulas $\left({ }_{i} D_{q}\right.$ denotes the derivative with respect to variable $i$ )

$$
\begin{gathered}
{[a(t-s)]^{(\alpha)}=a^{\alpha}(t-s)^{(\alpha)},} \\
{ }_{t} D_{q}(t-s)^{(\alpha)}=[\alpha]_{q}(t-s)^{(\alpha-1)}, \\
{ }_{x} D_{q} \int_{0}^{x} f(x, t) d_{q} t=\int_{0}^{x}{ }_{x} D_{q} f(x, t) d_{q} t+f(q x, x) .
\end{gathered}
$$

Remark 4. We note that if $\alpha \geq 0$ and $a \leq b \leq t$, then $(t-a)^{(\alpha)} \geq$ $(t-b)^{(\alpha)}[24]$.

Definition 5 (see [21]). Let $\alpha \geq 0$ and $f$ be a function defined on $[0,1]$. The fractional $q$-integral of the Riemann-Liuville type is $\left(I_{q}^{0} f\right)(x)=f(x)$ and

$$
\begin{array}{r}
\left(I_{q}^{\alpha} f\right)(x)=\frac{1}{\Gamma_{q}(\alpha)} \int_{0}^{x}(x-q t)^{(\alpha-1)} f(t) d_{q} t \\
\alpha>0, \quad x \in[0,1] .
\end{array}
$$

Definition 6 (see [23]). The fractional $q$-derivative of the Riemann-Liuville type of $\alpha \geq 0$ is defined by $\left(D_{q}^{0} f\right)(x)=$ $f(x)$ and

$$
\left(D_{q}^{\alpha} f\right)(x)=\left(D_{q}^{m} I_{q}^{m-\alpha} f\right)(x), \quad \alpha>0,
$$

where $m$ is the smallest integer greater than or equal to $\alpha$.

Lemma 7 (see $[21,23]$ ). Let $\alpha, \beta \geq 0$ and let $f$ be a function define on $[0,1]$. Then, the next formulas hold:
(1) $\left(I_{q}^{\beta} I_{q}^{\alpha} f\right)(x)=\left(I_{q}^{\alpha+\beta} f\right)(x)$,
(2) $\left(D_{q}^{\alpha} I_{q}^{\alpha} f\right)(x)=f(x)$.

Lemma 8 (see [24]). Let $\alpha>0$ and let $p$ be a positive integer. Then, the following equality holds:

$$
\begin{aligned}
\left(I_{q}^{\alpha} D_{q}^{p} f\right)(x)= & \left(D_{q}^{p} I_{q}^{\alpha} f\right)(x) \\
& -\sum_{k=0}^{p-1} \frac{x^{\alpha-p+k}}{\Gamma_{q}(\alpha+k-p+1)}\left(D_{q}^{k} f\right)(0) .
\end{aligned}
$$

Lemma 9. Let $y(t) \in C[0,1] \cap L^{1}[0,1]$ and $2<\alpha \leq 3$; then the BVP

$$
\begin{gathered}
\left(D_{q}^{\alpha} u\right)(t)+y(t)=0, \quad 0<t<1, \\
u(0)=u(1)=0, \quad\left(D_{q} u\right)(0)=0,
\end{gathered}
$$


has a unique solution

$$
u(t)=\int_{0}^{1} G(t, q s) y(s) d_{q} s
$$

where

$$
\begin{aligned}
& G(t, s) \\
& =\frac{1}{\Gamma_{q}(\alpha)} \begin{cases}(1-s)^{(\alpha-1)} t^{\alpha-1}-(t-s)^{(\alpha-1)}, & 0 \leq s \leq t \leq 1 \\
(1-s)^{(\alpha-1)} t^{\alpha-1}, & 0 \leq t \leq s \leq 1\end{cases}
\end{aligned}
$$

Proof. By Lemmas 7 and 8, we see that

$$
\begin{aligned}
\left(D_{q}^{\alpha} u\right)(t) & =-y(t) \\
& \Longleftrightarrow\left(I_{q}^{\alpha} D_{q}^{3} I_{q}^{3-\alpha} u\right)(t)=-\left(I_{q}^{\alpha} y\right)(t) \\
\Longleftrightarrow u(t)=c_{1} t^{\alpha-1}+c_{2} t^{\alpha-2} & \\
& +c_{3} t^{\alpha-3}-\frac{1}{\Gamma_{q}(\alpha)} \int_{0}^{t}(t-q s)^{(\alpha-1)} \\
& \times y(s) d_{q} s
\end{aligned}
$$

where $c_{1}, c_{2}$, and $c_{3}$ are some constants to be determined. Since $u(0)=0$, we must have $c_{3}=0$. Now, differentiating both sides of (22) and using (15), we obtain

$$
\begin{aligned}
\left(D_{q} u\right)(t)= & {[\alpha-1]_{q} c_{1} t^{\alpha-2}+[\alpha-2]_{q} c_{2} t^{\alpha-3} } \\
& -\frac{1}{\Gamma_{q}(\alpha)} \int_{0}^{t}[\alpha-1]_{q}(t-q s)^{(\alpha-2)} y(s) d_{q} s .
\end{aligned}
$$

Using $\left(D_{q} u\right)(0)=0$ and $u(1)=0$, we must set $c_{2}=0$, and

$$
c_{1}=\frac{1}{\Gamma_{q}(\alpha)} \int_{0}^{1}(1-q s)^{(\alpha-1)} y(s) d_{q} s .
$$

Finally, we obtain

$$
\begin{aligned}
u(t)= & \frac{t^{\alpha-1}}{\Gamma_{q}(\alpha)} \int_{0}^{1}(1-q s)^{(\alpha-1)} y(s) d_{q} s \\
& -\frac{1}{\Gamma_{q}(\alpha)} \int_{0}^{t}(t-q s)^{(\alpha-1)} y(s) d_{q} s \\
= & \int_{0}^{1} G(t, q s) y(s) d_{q} s .
\end{aligned}
$$

The proof is complete.

Lemma 10. Function $G$ defined above satisfies the following conditions:

(i) $G(t, q s)$ is a continuous function on $[0,1] \times[0,1]$;

(ii) $G(t, q s) \geq 0$ for $t, s \in[0,1]$.
Proof. (i) Obviously, $G(t, q s)$ is continuous on $[0,1] \times[0,1]$. (ii) Let

$$
g_{1}(t, s)=(1-s)^{(\alpha-1)} t^{\alpha-1}-(t-s)^{(\alpha-1)},
$$

$$
0 \leq s \leq t \leq 1
$$

$$
g_{2}(t, s)=(1-s)^{(\alpha-1)} t^{\alpha-1}, \quad 0 \leq t \leq s \leq 1 \text {. }
$$

It is clear that $g_{2}(t, q s) \geq 0$, for $t, s \in[0,1]$. Now, in view of Remark 4 , for $t \neq 0$

$$
\begin{aligned}
g_{1}(t, q s) & =(1-q s)^{(\alpha-1)} t^{\alpha-1}-(t-q s)^{(\alpha-1)} \\
& =t^{\alpha-1}\left[(1-q s)^{(\alpha-1)}-\left(1-\frac{q s}{t}\right)^{(\alpha-1)}\right] \\
& \geq t^{\alpha-1}\left[(1-q s)^{(\alpha-1)}-(1-q s)^{(\alpha-1)}\right]=0 .
\end{aligned}
$$

Therefore, $G(t, q s) \geq 0$. This proof is complete.

By $\mathscr{J}$ we denote the class of those functions $\beta:[0, \infty) \rightarrow$ $[0,1)$ satisfying the following condition; $\beta\left(t_{n}\right) \rightarrow 1$ implies $t_{n} \rightarrow 0$.

Theorem 11 (see $[31])$. Let $(X, \leq)$ be a partially ordered set and suppose that there exists a metric $d$ in $X$ such that $(X, d)$ is a complete metric space. Let $T: X \rightarrow X$ be a nondecreasing mapping such that there exists an element $x_{0} \in X$ with $x_{0} \leq$ $T x_{0}$. Suppose that there exists $\beta \in \mathcal{J}$ such that

$$
\begin{array}{r}
d(T x, T y) \leq \beta(d(x, y)) \cdot d(x, y) \\
\text { for } x, y \in X \text { with } x \geq y .
\end{array}
$$

Assume that either $T$ is continuous or $X$ is such that

$$
\begin{aligned}
& \text { if }\left\{x_{n}\right\} \text { is a nondecreasing sequence in } X \\
& \text { such that } x_{n} \longrightarrow x \text { then } x_{n} \leq x \quad \forall n \in N .
\end{aligned}
$$

Besides if

$$
\begin{array}{r}
\text { for each } x, y \in X \text { there exists } z \in X \\
\text { which is comparable to } x \text { and } y,
\end{array}
$$

then $T$ has a unique fixed point.

Let $C[0,1]=\{x:[0,1] \rightarrow R$, continuous $\}$ be the Banach space with the classic metric given by $d(x, y)=$ $\sup _{0 \leq t \leq 1}\{|x(t)-y(t)|\}$.

Notice that this space can be equipped with a partial order given by

$$
x, y \in C[0,1], \quad x \leq y \Longleftrightarrow x(t) \leq y(t), \quad \text { for } t \in[0,1] .
$$

In [32], it is proved that $(C[0,1], \leq)$ satisfies condition (29) of Theorem 11. Moreover, for $x, y \in C[0,1]$, as the function $\max (x, y) \in C[0,1],(C[0,1], \leq)$ satisfies condition (30). 


\section{Main Result}

In this section, we will consider the question of positive solutions for BVP (3). At first, we prove some lemmas required for the main result.

Lemma 12. Let $0<\sigma<1,2<\alpha \leq 3$ and $F:(0,1] \rightarrow$ $R$ is a continuous function with $\lim _{t \rightarrow 0^{+}} F(t)=\infty$. Suppose that $t^{\sigma} F(t)$ is a continuous function on $[0,1]$. Then the function defined by

$$
H(t)=\int_{0}^{1} G(t, q s) F(s) d_{q} s
$$

is continuous on $[0,1]$, where $G(t, s)$ is Green function be given in Lemma 9.

Proof. We will divide the proof into three parts.

Case $1\left(t_{0}=0\right)$. First, $H(0)=0$. Since $t^{\sigma} F(t)$ is continuous on $[0,1]$, we can find a positive constant $M$ such that $\left|t^{\sigma} F(t)\right| \leq$ $M$ for any $t \in[0,1]$. Thus,

$$
\begin{aligned}
& |H(t)-H(0)| \\
& =|H(t)| \\
& =\left|\int_{0}^{1} G(t, q s) F(s) d_{q} s\right| \\
& =\left|\int_{0}^{1} G(t, q s) s^{-\sigma} s^{\sigma} F(s) d_{q} s\right| \\
& =\mid \int_{0}^{t} \frac{(1-q s)^{(\alpha-1)} t^{\alpha-1}-(t-q s)^{(\alpha-1)}}{\Gamma_{q}(\alpha)} s^{-\sigma} s^{\sigma} F(s) d_{q} s \\
& +\int_{t}^{1} \frac{(1-q s)^{(\alpha-1)} t^{\alpha-1}}{\Gamma_{q}(\alpha)} s^{-\sigma} s^{\sigma} F(s) d_{q} s \\
& =\mid \int_{0}^{1} \frac{(1-q s)^{(\alpha-1)} t^{\alpha-1}}{\Gamma_{q}(\alpha)} s^{-\sigma} s^{\sigma} F(s) d_{q} s \\
& -\int_{0}^{t} \frac{(t-q s)^{(\alpha-1)}}{\Gamma_{q}(\alpha)} s^{-\sigma} s^{\sigma} F(s) d_{q} s \\
& \leq M \int_{0}^{1} \frac{(1-q s)^{(\alpha-1)} t^{\alpha-1}}{\Gamma_{q}(\alpha)} s^{-\sigma} d_{q} s \\
& +M \int_{0}^{t} \frac{(t-q s)^{(\alpha-1)}}{\Gamma_{q}(\alpha)} s^{-\sigma} d_{q} s \\
& =\frac{M t^{\alpha-1}}{\Gamma_{q}(\alpha)}\left[\int_{0}^{1}(1-q s)^{(\alpha-1)} s^{-\sigma} d_{q} s\right. \\
& \left.+\int_{0}^{t}\left(1-\frac{q s}{t}\right)^{(\alpha-1)} s^{-\sigma} d_{q} s\right] .
\end{aligned}
$$

For $\int_{0}^{t}(1-(q s / t))^{(\alpha-1)} s^{-\sigma} d_{q} s$, let $u=s / t$; then we obtain

$$
\int_{0}^{t}\left(1-\frac{q s}{t}\right)^{(\alpha-1)} s^{-\sigma} d_{q} s=t^{1-\sigma} \int_{0}^{1}(1-q u)^{(\alpha-1)} u^{-\sigma} d_{q} u .
$$

Hence,

$$
\begin{aligned}
|H(t)| \leq & \frac{M t^{\alpha-1}}{\Gamma_{q}(\alpha)} \int_{0}^{1}(1-q s)^{(\alpha-1)} s^{-\sigma} d_{q} s \\
& +\frac{M t^{\alpha-\sigma}}{\Gamma_{q}(\alpha)} \int_{0}^{1}(1-q u)^{(\alpha-1)} u^{-\sigma} d_{q} u \\
= & \left(\frac{M t^{\alpha-1}}{\Gamma_{q}(\alpha)}+\frac{M t^{\alpha-\sigma}}{\Gamma_{q}(\alpha)}\right) \beta_{q}(1-\sigma, \alpha),
\end{aligned}
$$

where $\beta_{q}$ denotes the $q$-beta function.

When $t \rightarrow 0$, we see that $H(t) \rightarrow H(0)$; that is $H(t)$ is continuous at $t_{0}=0$.

Case $2\left(t_{0} \in(0,1)\right)$. We should prove $H\left(t_{n}\right) \rightarrow H\left(t_{0}\right)$ when $t_{n} \rightarrow t_{0}$. Without loss of generality, we consider $t_{n}>t_{0}$ (it is the same argument for $\left.t_{n}<t_{0}\right)$. In fact,

$$
\begin{aligned}
& \left|H\left(t_{n}\right)-H\left(t_{0}\right)\right| \\
& =\mid \int_{0}^{1} \frac{t_{n}^{\alpha-1}(1-q s)^{(\alpha-1)}}{\Gamma_{q}(\alpha)} s^{-\sigma} s^{\sigma} F(s) d_{q} s \\
& -\int_{0}^{t_{n}} \frac{\left(t_{n}-q s\right)^{(\alpha-1)}}{\Gamma_{q}(\alpha)} s^{-\sigma} s^{\sigma} F(s) d_{q} s \\
& -\int_{0}^{1} \frac{t_{0}^{\alpha-1}(1-q s)^{(\alpha-1)}}{\Gamma_{q}(\alpha)} s^{-\sigma} s^{\sigma} F(s) d_{q} s \\
& +\int_{0}^{t_{0}} \frac{\left(t_{0}-q s\right)^{(\alpha-1)}}{\Gamma_{q}(\alpha)} s^{-\sigma} s^{\sigma} F(s) d_{q} s \\
& =\mid \int_{0}^{1} \frac{\left(t_{n}^{\alpha-1}-t_{0}^{\alpha-1}\right)(1-q s)^{(\alpha-1)}}{\Gamma_{q}(\alpha)} s^{-\sigma} s^{\sigma} F(s) d_{q} s \\
& -\int_{0}^{t_{0}} \frac{\left(t_{n}-q s\right)^{(\alpha-1)}-\left(t_{0}-q s\right)^{(\alpha-1)}}{\Gamma_{q}(\alpha)} s^{-\sigma} s^{\sigma} F(s) d_{q} s \\
& -\int_{t_{0}}^{t_{n}} \frac{\left(t_{n}-q s\right)^{(\alpha-1)}}{\Gamma_{q}(\alpha)} s^{-\sigma} s^{\sigma} F(s) d_{q} s \\
& \leq \frac{M\left(t_{n}^{\alpha-1}-t_{0}^{\alpha-1}\right)}{\Gamma_{q}(\alpha)} \int_{0}^{1}(1-q s)^{(\alpha-1)} s^{-\sigma} d_{q} s \\
& +\frac{M}{\Gamma_{q}(\alpha)} \int_{0}^{t_{0}}\left(\left(t_{n}-q s\right)^{(\alpha-1)}-\left(t_{0}-q s\right)^{(\alpha-1)}\right) s^{-\sigma} d_{q} s
\end{aligned}
$$




$$
\begin{aligned}
& +\frac{M}{\Gamma_{q}(\alpha)} \int_{t_{0}}^{t_{n}}\left(t_{n}-q s\right)^{(\alpha-1)} s^{-\sigma} d_{q} s \\
= & \frac{M}{\Gamma_{q}(\alpha)} \beta_{q}(1-\sigma, \alpha)\left(t_{n}^{\alpha-1}-t_{0}^{\alpha-1}\right)+\frac{M}{\Gamma_{q}(\alpha)}\left(a_{n}+b_{n}\right),
\end{aligned}
$$

where

$$
\begin{gathered}
a_{n}=\int_{0}^{t_{0}}\left(\left(t_{n}-q s\right)^{(\alpha-1)}-\left(t_{0}-q s\right)^{(\alpha-1)}\right) s^{-\sigma} d_{q} s, \\
b_{n}=\int_{t_{0}}^{t_{n}}\left(t_{n}-q s\right)^{(\alpha-1)} s^{-\sigma} d_{q} s .
\end{gathered}
$$

When $n \rightarrow \infty$, we verify $a_{n} \rightarrow 0$.

$$
\text { As } t_{n} \rightarrow t_{0} \text {, then }\left(\left(t_{n}-q s\right)^{(\alpha-1)}-\left(t_{0}-q s\right)^{(\alpha-1)}\right) s^{-\sigma} \rightarrow 0,
$$
when $n \rightarrow \infty$. Moreover,

$$
\begin{gathered}
\left(\left(t_{n}-q s\right)^{(\alpha-1)}-\left(t_{0}-q s\right)^{(\alpha-1)}\right) s^{-\sigma} \leq 2 s^{-\sigma} \\
\int_{0}^{1} 2 s^{-\sigma} d_{q} s=\left.\frac{2}{[1-\sigma]_{q}} s^{1-\sigma}\right|_{0} ^{1}=\frac{2}{[1-\sigma]_{q}}<\infty .
\end{gathered}
$$

We have $\left(\left(t_{n}-q s\right)^{(\alpha-1)}-\left(t_{0}-q s\right)^{(\alpha-1)}\right) s^{-\sigma}$ converges pointwise to the zero function and $\mid\left(t_{n}-q s\right)^{(\alpha-1)}-\left(t_{0}-\right.$ $q s)^{(\alpha-1)} \mid s^{-\sigma}$ is bounded by a function belonging to $L^{1}[0,1]$, by Lebesgue's dominated convergence theorem $a_{n} \rightarrow 0$ when $n \rightarrow \infty$.

Now, we prove $b_{n} \rightarrow 0$ when $n \rightarrow \infty$.

In fact, as

$$
\begin{aligned}
b_{n} & =\int_{t_{0}}^{t_{n}}\left(t_{n}-q s\right)^{(\alpha-1)} s^{-\sigma} d_{q} s \\
& \leq \int_{t_{0}}^{t_{n}} s^{-\sigma} d_{q} s=\left.\frac{s^{1-\sigma}}{[1-\sigma]_{q}}\right|_{t_{0}} ^{t_{n}} \\
& =\frac{1}{[1-\sigma]_{q}}\left(t_{n}^{1-\sigma}-t_{0}^{1-\sigma}\right),
\end{aligned}
$$

and taking into account that $t_{n} \rightarrow t_{0}$, we get $b_{n} \rightarrow 0$ when $n \rightarrow \infty$.

Above all, we obtain $\left|H\left(t_{n}\right)-H\left(t_{0}\right)\right| \rightarrow 0$, when $n \rightarrow \infty$.

Case $3\left(t_{0}=1\right)$. It is easy to check that $H(1)=0$ and $H(t)$ is continuous at $t_{0}=1$. The proof is the same as the proof of Case 1.

Lemma 13. Suppose that $0<\sigma<1$. Then,

$$
\max _{0 \leq t \leq 1} \int_{0}^{1} G(t, q s) s^{-\sigma} d_{q} s=\frac{A^{\alpha-1}-A^{\alpha-\sigma}}{\Gamma_{q}(\alpha)} \beta_{q}(1-\sigma, \alpha),
$$

where $A=((\alpha-1) /(\alpha-\sigma))^{1 /(1-\sigma)}$.
Proof.

$$
\begin{aligned}
& \int_{0}^{1} G(t, q s) s^{-\sigma} d_{q} s \\
& \quad=\int_{0}^{t} \frac{(1-q s)^{(\alpha-1)} t^{\alpha-1}-(t-q s)^{(\alpha-1)}}{\Gamma_{q}(\alpha)} s^{-\sigma} d_{q} s
\end{aligned}
$$$$
+\int_{t}^{1} \frac{(1-q s)^{(\alpha-1)} t^{\alpha-1}}{\Gamma_{q}(\alpha)} s^{-\sigma} d_{q} s
$$$$
=\int_{0}^{1} \frac{(1-q s)^{(\alpha-1)} t^{\alpha-1}}{\Gamma_{q}(\alpha)} s^{-\sigma} d_{q} s
$$$$
-\int_{0}^{t} \frac{(t-q s)^{(\alpha-1)}}{\Gamma_{q}(\alpha)} s^{-\sigma} d_{q} s
$$

$$
\begin{aligned}
= & \frac{t^{\alpha-1}}{\Gamma_{q}(\alpha)} \int_{0}^{1}(1-q s)^{(\alpha-1)} s^{-\sigma} d_{q} s \\
& -\frac{t^{\alpha-\sigma}}{\Gamma_{q}(\alpha)} \int_{0}^{1}(1-q s)^{(\alpha-1)} s^{-\sigma} d_{q} u \\
= & \frac{t^{\alpha-1}-t^{\alpha-\sigma}}{\Gamma_{q}(\alpha)} \beta_{q}(1-\sigma, \alpha) .
\end{aligned}
$$

Let $g(t)=t^{\alpha-1}-t^{\alpha-\sigma}, t \in[0,1]$.

Since $g^{\prime}(t)=(\alpha-1) t^{\alpha-2}-(\alpha-\sigma) t^{\alpha-\sigma-1}$, let $g^{\prime}(t)=0$; we can get $g(t)$ has a maximum at the point $t_{0}=A=((\alpha-$ $1) /(\alpha-\sigma))^{1 /(1-\sigma)}$.

Hence,

$$
\max _{0 \leq t \leq 1} \int_{0}^{1} G(t, q s) s^{-\sigma} d_{q} s=\frac{A^{\alpha-1}-A^{\alpha-\sigma}}{\Gamma_{q}(\alpha)} \beta_{q}(1-\sigma, \alpha) .
$$

For the convenience, we denote $\max _{0 \leq t \leq 1} \int_{0}^{1} G(t$, $q s) s^{-\sigma} d_{q} s$ by $K$

Next, we denote the class of functions $\phi:[0, \infty) \rightarrow$ $[0, \infty)$ by $\mathscr{A}$ satisfying

(i) $\phi$ is nondecreasing;

(ii) $\phi(x)<x$ for any $x>0$;

(iii) $\beta(x)=\phi(x) / x \in \mathscr{J}$, where $\mathscr{J}$ is the class of functions appearing in Theorem 11 .

We give our main result as follows.

Theorem 14. Let $0<\sigma<1,2<\alpha \leq 3, f:[0,1] \times[0, \infty) \rightarrow$ $[0, \infty)$ is continuous and $\lim _{t \rightarrow 0^{+}} f(t, \cdot)=\infty$, and $t^{\sigma} f(t, y)$ is a continuous function on $[0,1] \times[0, \infty)$. Assume that there exists $0<\lambda \leq 1 / K$ such that for $x, y \in[0, \infty)$ with $y \geq x$ and $t \in[0,1]$,

$$
0 \leq t^{\sigma}(f(t, y)-f(t, x)) \leq \lambda \phi(y-x),
$$

where $\phi \in \mathscr{A}$. Then the BVP (3) has a unique positive solution (i.e., $x(t)>0$ for $t \in(0,1)$ ). 
Proof. We define the cone $P$ by

$$
P=\{u \in C[0,1]: u(t) \geq 0\} .
$$

It is clear that $P$ is a complete metric space as $P$ is a closed set of $C[0,1]$. It is also easy to check that $P$ satisfies conditions (29) and (30) of Theorem 11.

We define the operator $T$ by

$$
\begin{aligned}
(T u)(t) & =\int_{0}^{1} G(t, q s) f(s, u(s)) d_{q} s \\
& =\int_{0}^{1} G(t, q s) s^{-\sigma} s^{\sigma} f(s, u(s)) d_{q} s .
\end{aligned}
$$

In view of Lemma $12, T u \in C[0,1]$. Moreover, it follows from the nonnegativeness of $G(t, q s)$ and $t^{\sigma} f(t, y)$ that $T u \in P$ for $u \in P$. Thus, $T: P \rightarrow P$.

Next, we will prove that assumptions in Theorem 11 are satisfied.

First, for $u \geq v$, we have

$$
\begin{aligned}
(T u)(t) & =\int_{0}^{1} G(t, q s) f(s, u(s)) d_{q} s \\
& =\int_{0}^{1} G(t, q s) s^{-\sigma} s^{\sigma} f(s, u(s)) d_{q} s \\
& \geq \int_{0}^{1} G(t, q s) s^{-\sigma} s^{\sigma} f(s, v(s)) d_{q} s \\
& =(T v)(t) .
\end{aligned}
$$

Hence, the operator $T$ is nondecreasing. Besides, for $u \geq v$ and $u \neq v$,

$$
\begin{aligned}
& d(T u, T v) \\
& =\max _{t \in[0,1]}|(T u)(t)-(T v)(t)| \\
& =\max _{t \in[0,1]}((T u)(t)-(T v)(t)) \\
& =\max _{t \in[0,1]} \int_{0}^{1} G(t, q s)(f(s, u(s))-f(s, v(s))) d_{q} s \\
& =\max _{t \in[0,1]} \int_{0}^{1} G(t, q s) s^{-\sigma} s^{\sigma}(f(s, u(s))-f(s, v(s))) d_{q} s \\
& \leq \max _{t \in[0,1]} \int_{0}^{1} G(t, q s) s^{-\sigma} \lambda \phi(u(s)-v(s)) d_{q} s .
\end{aligned}
$$

Since $\phi$ is nondecreasing and $u(s)-v(s) \leq d(u, v)$,

$$
\begin{aligned}
d(T u, T v) & \leq \max _{t \in[0,1]} \int_{0}^{1} G(t, q s) s^{-\sigma} \lambda \phi(d(u, v)) d_{q} s \\
& =\lambda \phi(d(u, v)) \max _{t \in[0,1]} \int_{0}^{1} G(t, q s) s^{-\sigma} d_{q} s \\
& =\lambda \phi(d(u, v)) K .
\end{aligned}
$$

Moreover, when $0<\lambda \leq 1 / K$, we get

$$
\begin{aligned}
d(T u, T v) & \leq \phi(d(u, v)) \\
& =\frac{\phi(d(u, v))}{d(u, v)} \cdot d(u, v) \\
& =\beta(d(u, v)) \cdot d(u, v) .
\end{aligned}
$$

Obviously, the last inequality is satisfied for $u=v$.

Taking into account that the zero function satisfies $0 \leq T_{0}$, in view of Theorem 11, the operator $T$ has a unique fixed point $x(t)$ in $P$.

At last, we will prove $x(t)$ is a positive solution. We assume that there exists $0<t_{1}<1$ such that $x\left(t_{1}\right)=0$. Since $x(t)$ of problem (3) is a fixed point of the operator $T$, we have

$$
\begin{gathered}
x(t)=\int_{0}^{1} G(t, q s) f(s, x(s)) d_{q} s, \quad \text { for } 0<t<1, \\
x\left(t_{1}\right)=\int_{0}^{1} G\left(t_{1}, q s\right) f(s, x(s)) d_{q} s=0 .
\end{gathered}
$$

For the nonnegative character of $G(t, q s)$ and $f(s, x)$, the last relation gives

$$
G\left(t_{1}, q s\right) f(s, x(s))=0 \quad \text { a.e. }(s) .
$$

$f$ is continuous and $\lim _{t \rightarrow 0^{+}} f(t, \cdot)=\infty$; then for $M>0$, we can find $\delta>0$, and, for $s \in[0,1] \cap(0, \delta)$, we have $f(s, 0)>M$. It is clear that $[0,1] \cap(0, \delta) \subset\{s \in[0,1]: f(s, x(s))>M\}$ and $\mu([0,1] \cap(0, \delta))>0$, where $\mu$ is the Lebesgue measure on $[0,1]$. That is to say, $G\left(t_{1}, q s\right) f(s, x(s))=0$ a.e. $(s)$. This is a contradiction because $G\left(t_{1}, q s\right)$ is a rational function in $s$.

Therefore, $x(t)>0$ for $t \in(0,1)$.

The proof is complete.

\section{Example}

Consider the following singular BVP:

$$
\begin{gathered}
D_{1 / 2}^{5 / 2} u(t)+\frac{\lambda\left(t^{2}+1\right) \ln (1+u(t))}{t^{1 / 2}}=0, \quad 0<t<1, \lambda>0, \\
u(0)=u(1)=0, \quad\left(D_{1 / 2} u\right)(0)=0 .
\end{gathered}
$$

Here, $\alpha=2.5, q=1 / 2, \sigma=1 / 2$, and $f(t, u)=\lambda\left(t^{2}+\right.$ 1) $\ln (1+u(t)) / t^{1 / 2}$ for $(t, u) \in[0,1] \times[0, \infty)$. Notice that $f$ is continuous in $[0,1] \times[0, \infty)$ and $\lim _{t \rightarrow 0^{+}} f(t, \cdot)=\infty$.

At first, we define $\phi$ by

$$
\phi:[0, \infty) \longrightarrow[0, \infty), \quad \phi(x)=\ln (1+x) .
$$

It is clear that $\phi(x)=\ln (1+x)$ is a nondecreasing function; for $u \geq v$, we can get

$$
\phi(u)-\phi(v) \geq 0 .
$$

Moreover, for $u \geq v, \phi$ also satisfies

$$
\phi(u)-\phi(v) \leq \phi(u-v) .
$$


In fact, when $u \geq v$,

$$
\begin{aligned}
\phi(u-v)-(\phi(u)-\phi(v))= & \ln (1+u-v) \\
& -(\ln (1+u)-\ln (1+v)) \\
= & \ln \frac{(1+u-v)(1+v)}{(1+u)} \\
= & \ln \left(1+\frac{(u-v) v}{1+u}\right) \geq 0,
\end{aligned}
$$

equivalently

$$
\phi(u)-\phi(v) \leq \phi(u-v) .
$$

Above all, $0 \leq \phi(u)-\phi(v) \leq \phi(u-v)$ for $u \geq v$.

Second, for $u \geq v$ and $t \in[0,1]$, we have

$$
\begin{aligned}
0 & \leq t^{1 / 2}(f(t, u)-f(t, v)) \\
& =\lambda\left(t^{2}+1\right)[\ln (1+u)-\ln (1+v)] \\
& \leq \lambda\left(t^{2}+1\right) \ln (1+u-v) \\
& \leq 2 \lambda \ln (1+u-v)
\end{aligned}
$$

that is, $f$ satisfies assumptions of Theorem 14 .

Third, we should prove $\phi(x)$ belongs to $\mathscr{A}$. By elemental calculus, it is easy to check that $\phi$ is nondecreasing and $\phi(x)<$ $x$, for $x>0$.

In order to prove $\beta(x)=\phi(x) / x \in \mathcal{J}$, we notice that if $\beta\left(t_{n}\right) \rightarrow 1$, then the sequence $\left(t_{n}\right)$ is a bounded sequence because in contrary case, that is, $t_{n} \rightarrow \infty$, we get

$$
\beta\left(t_{n}\right)=\frac{\ln \left(1+t_{n}\right)}{t_{n}} \longrightarrow 0 .
$$

Now, we assume that $t_{n} \nrightarrow 0$, and then we find $\varepsilon>0$ such that for each $n \in N$ there exists $\rho_{n} \geq n$ with $t_{\rho_{n}} \geq \varepsilon$.

Since $\left(t_{n}\right)$ is a bounded sequence, we can find a subsequence $\left(t_{k_{n}}\right)$ of $\left(t_{\rho_{n}}\right)$ with $t_{k_{n}} \rightarrow a$, for certain $a \in[0,1)$. When $\beta\left(t_{n}\right) \rightarrow 1$, it implies that

$$
\beta\left(t_{k_{n}}\right)=\frac{\ln \left(1+t_{k_{n}}\right)}{t_{k_{n}}} \longrightarrow 1 .
$$

and, as the unique solution of $\ln (1+x)=x$ is $x_{0}=0$, we deduce that $a=0$. Therefore, $t_{k_{n}} \rightarrow 0$ and this contradicts the fact that $t_{k_{n}} \geq \varepsilon$ for every $n \in N$.

Thus, $t_{n} \rightarrow 0$ and this proves that $\beta \in \mathcal{J}$.

Finally, in view of Theorem 14,

$$
\begin{aligned}
2 \lambda & \leq \frac{1}{K}=\frac{1}{\left(\left((1 / 4)^{3 / 2}-(1 / 4)^{1 / 2}\right) / \Gamma_{1 / 2}(3 / 2)\right) \cdot \beta_{1 / 2}(1 / 2,3 / 2)} \\
& \approx 10.96511985 ;
\end{aligned}
$$

that is, when $\lambda \leq 5.48256$, boundary value problem (52) has a unique positive solution.

\section{Conflict of Interests}

The authors declare that there is no conflict of interests regarding the publication of this paper.

\section{Acknowledgments}

This paper is supported by the Natural Science Foundation of China (11201112), the Natural Science Foundation of Hebei Province (A2013208147), (A2014208152), and (A2015208114), and the Foundation of Hebei Education Department (Z2014062).

\section{References}

[1] Z. Bai and H. Lü, "Positive solutions for boundary value problem of nonlinear fractional differential equation," Journal of Mathematical Analysis and Applications, vol. 311, no. 2, pp. 495-505, 2005.

[2] L. M. B. C. Campos, "On the solution of some simple fractional differential equations," International Journal of Mathematics and Mathematical Sciences, vol. 13, no. 3, pp. 481-496, 1990.

[3] D. Delbosco and L. Rodino, "Existence and uniqueness for a nonlinear fractional differential equation," Journal of Mathematical Analysis and Applications, vol. 204, no. 2, pp. 609-625, 1996.

[4] A. A. Kilbas and J. J. Trujillo, "Differential equations of fractional order: methods, results and problems-I," Applicable Analysis, vol. 78, no. 1-2, pp. 153-192, 2001.

[5] C. F. Li, X. N. Luo, and Y. Zhou, "Existence of positive solutions of the boundary value problem for nonlinear fractional differential equations," Computers \& Mathematics with Applications, vol. 59, no. 3, pp. 1363-1375, 2010.

[6] Y. Ling and S. S. Ding, "A class of analytic functions defined by fractional derivation," Journal of Mathematical Analysis and Applications, vol. 186, no. 2, pp. 504-513, 1994.

[7] K. S. Miller and B. Ross, An Introduction to the Fractional Calculus and Fractional Differential Equations, John Wiley \& Sons, New York, NY, USA, 1993.

[8] T. Qiu and Z. Bai, "Existence of positive solutions for singular fractional differential equations," Electronic Journal of Differential Equations, vol. 146, pp. 1-9, 2008.

[9] S. G. Samko, A. A. Kilbas, and O. I. Marichev, Fractional Integral and Derivative. Theory and Applications, Gordon and Breach Science Publishers, Yverdon, Switzerland, 1993.

[10] S. Zhang, "The existence of a positive solution for a nonlinear fractional differential equation," Journal of Mathematical Analysis and Applications, vol. 252, no. 2, pp. 804-812, 2000.

[11] Y. Zhou and F. Jiao, "Nonlocal Cauchy problem for fractional evolution equations," Nonlinear Analysis: Real World Applications, vol. 11, no. 5, pp. 4465-4475, 2010.

[12] V. Kac and P. Cheung, Quantum Calculus, Springer, New York, NY, USA, 2002.

[13] F. H. Jackson, " $q$-difference equations," The American Journal of Mathematics, vol. 32, no. 4, pp. 305-314, 1910.

[14] R. D. Carmichael, "The general theory of linear $q$-difference equations," The American Journal of Mathematics, vol. 34, no. 2, pp. 147-168, 1912.

[15] T. E. Mason, "On properties of the solutions of linear $q$ difference equations with entire function coefficients," The 
American Journal of Mathematics, vol. 37, no. 4, pp. 439-444, 1915.

[16] C. R. Adams, "On the linear ordinary $q$-difference equation," Annals of Mathematics: Second Series, vol. 30, no. 1-4, pp. 195205, 1928.

[17] D. N. Page, "Information in black hole radiation," Physical Review Letters, vol. 71, no. 23, pp. 3743-3746, 1993.

[18] B. Ahmad and S. K. Ntouyas, "Boundary value problems for $q$ difference inclusions," Abstract and Applied Analysis, vol. 2011, Article ID 292860, 15 pages, 2011.

[19] C. L. Yu and J. F. Wang, "Existence of solutions for nonlinear second-order $q$-difference equations with first-order $q$ derivatives," Advances in Difference Equations, vol. 2013, article 124, 2013.

[20] W. A. Al-Salam, "Some fractional $q$-integrals and q-derivatives," Proceedings of the Edinburgh Mathematical Society: Series II, vol. 15, pp. 135-140, 1966.

[21] R. P. Agarwal, "Certain fractional q-integrals and q-derivatives," Proceedings of the Cambridge Philosophical Society, vol. 66, pp. 365-370, 1969.

[22] P. M. Rajković, S. D. Marinković, and M. S. Stanković, "Fractional integrals and derivatives in q-calculus," Applicable Analysis and Discrete Mathematics, vol. 1, no. 1, pp. 311-323, 2007.

[23] F. M. Atici and P. W. Eloe, "Fractional q-calculus on a time scale," Journal of Nonlinear Mathematical Physics, vol. 14, no. 3, pp. 333-344, 2007.

[24] R. A. Ferreira, "Nontrivial solutions for fractional q-difference boundary value problems," Electronic Journal of Qualitative Theory of Differential Equations, vol. 70, pp. 1-10, 2010.

[25] R. A. C. Ferreira, "Positive solutions for a class of boundary value problems with fractional q-differences," Computers \& Mathematics with Applications, vol. 61, no. 2, pp. 367-373, 2011.

[26] M. El-Shahed and F. M. Al-Askar, "Positive solutions for boundary value problem of nonlinear fractional $q$-difference equation," ISRN Mathematical Analysis, vol. 2011, Article ID 385459, 12 pages, 2011.

[27] J. R. Graef and L. Kong, "Positive solutions for a class of higher order boundary value problems with fractional q-derivatives," Applied Mathematics and Computation, vol. 218, no. 19, pp. 9682-9689, 2012.

[28] B. Ahmad, S. K. Ntouyas, and I. K. Purnaras, "Existence results for nonlocal boundary value problems of nonlinear fractional $q$-difference equations Difference equations: new trends and applications in biology, medicine and biotechnology," Advances in Difference Equations, vol. 2012, article 140, 2012.

[29] C. Yu and J. Wang, "Positive solutions of nonlocal boundary value problem for high-order nonlinear fractional $q$-difference equations," Abstract and Applied Analysis, vol. 2013, Article ID 928147, 9 pages, 2013.

[30] J. Caballero, J. Harjani, and K. Sadarangani, "Positive solutions for a class of singular fractional boundary value problems," Computers \& Mathematics with Applications, vol. 62, no. 3, pp. 1325-1332, 2011.

[31] A. Amini-Harandi and H. Emani, "A fixed point theorem for contraction type maps in partially ordered metric spaces and application to ordinary differential equations," Nonlinear Analysis: Theory, Methods \& Applications, vol. 72, no. 5, pp. 2238-2242, 2010.

[32] J. J. Nieto and R. Rodríguez-López, "Contractive mapping theorems in partially ordered sets and applications to ordinary differential equations," Order, vol. 22, no. 3, pp. 223-239, 2005. 


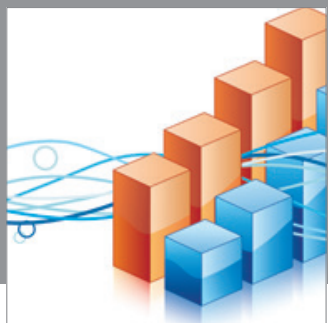

Advances in

Operations Research

mansans

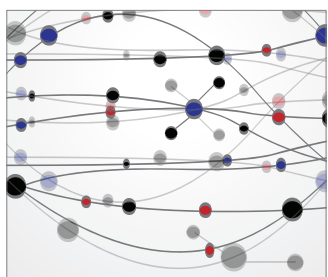

The Scientific World Journal
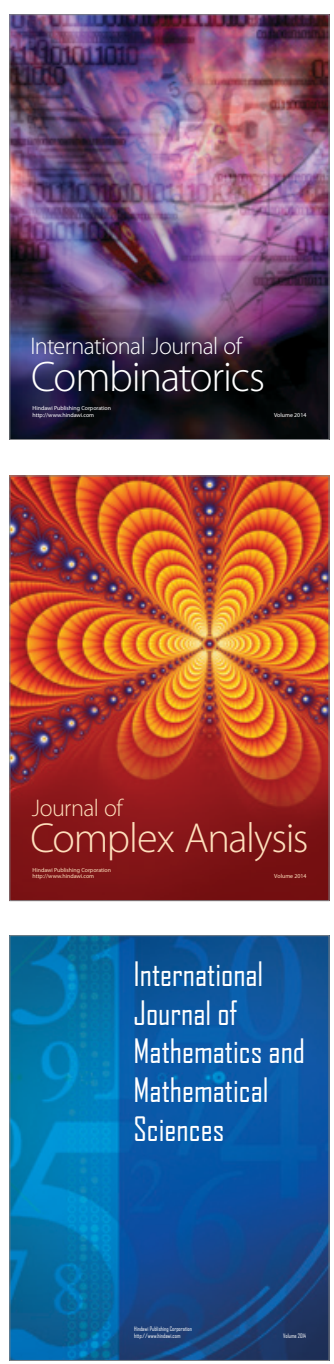
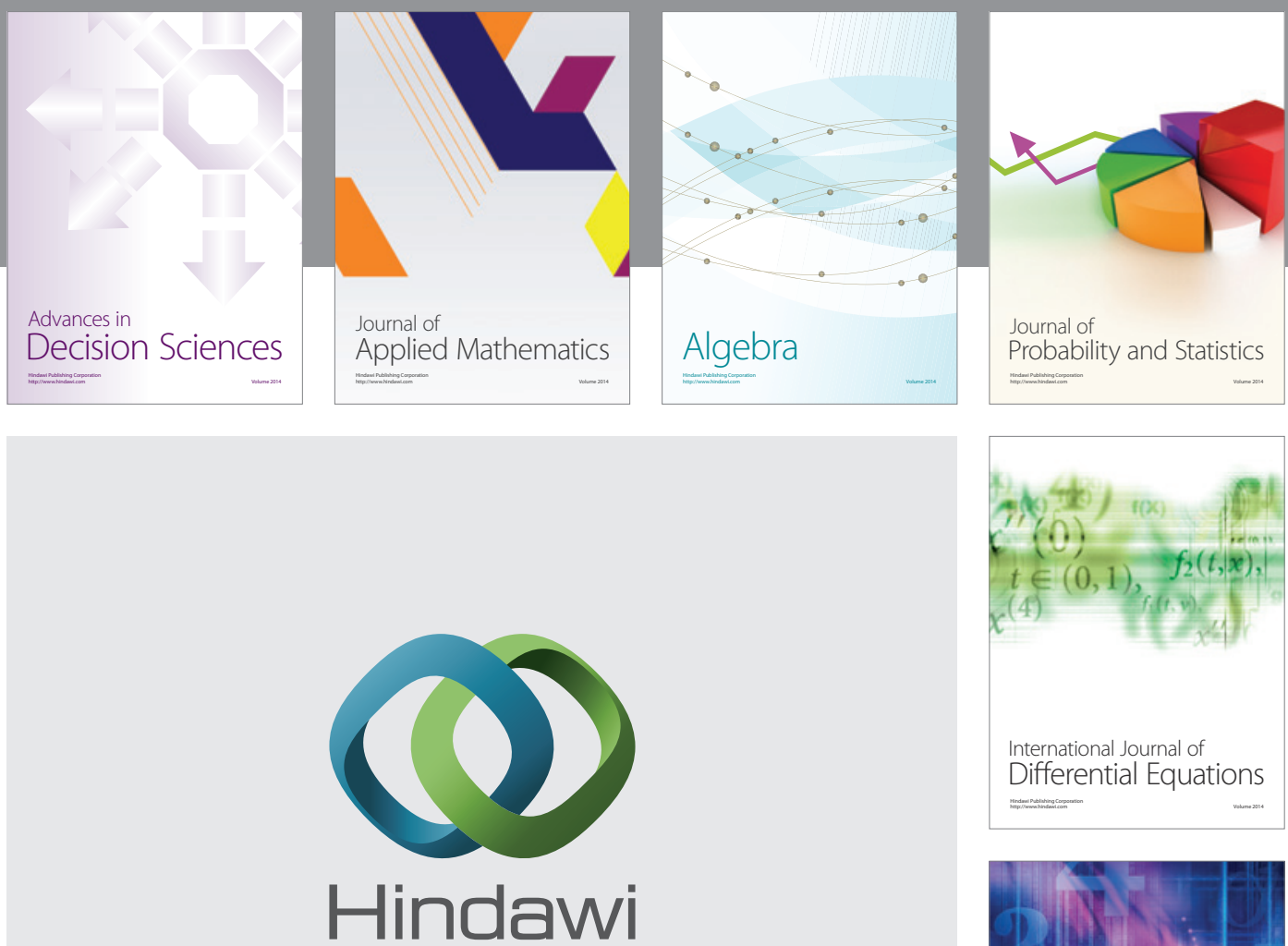

Submit your manuscripts at http://www.hindawi.com
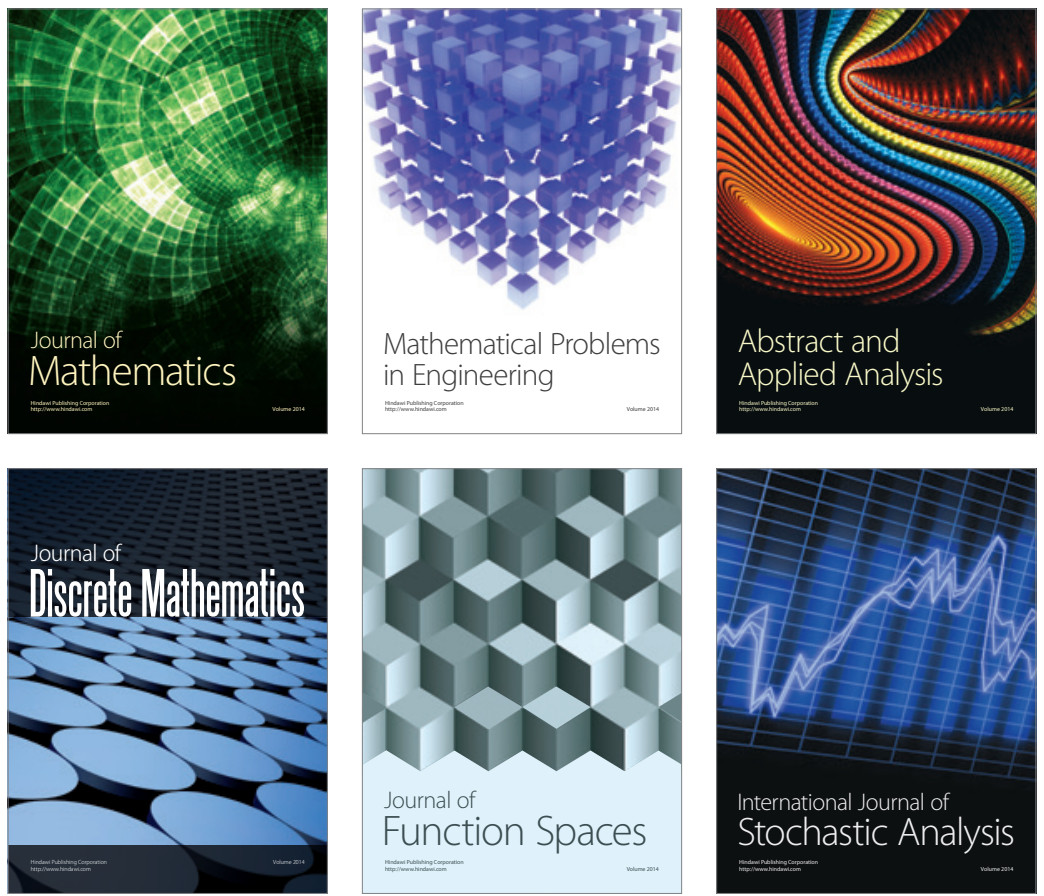

Journal of

Function Spaces

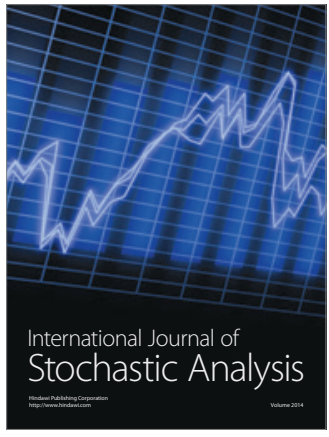

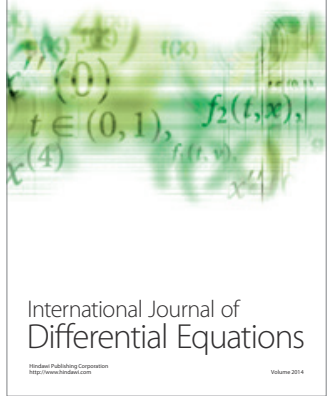
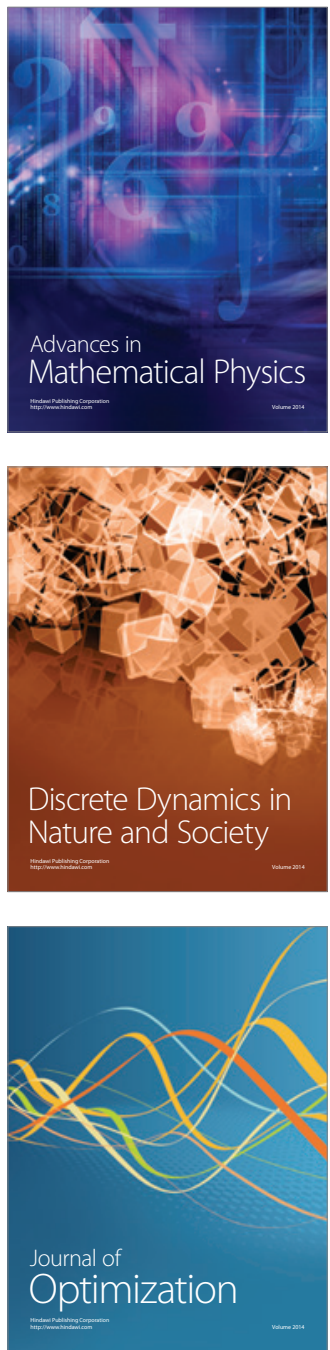